\title{
Le professeur nouveau est arrivé
}

Entretien avec Nicole Gaudelet, Inspectrice de l'Éducation nationale, Inspection académique de l'Essonne, Evry, France

The new teacher has appeared. Interview with Nicole Gaudelet

El docente " nuevo " ha llegado. Entrevista con Nicole Gaudelet

Pierre-Louis Gauthier

\section{OpenEdition}

Journals

Édition électronique

URL : http://journals.openedition.org/ries/2827

DOI : $10.4000 /$ ries. 2827

ISSN : 2261-4265

Éditeur

Centre international d'études pédagogiques

Édition imprimée

Date de publication : 1 mars 1999

Pagination : 129-132

ISBN : 9771254459005

ISSN : $1254-4590$

\section{Référence électronique}

Pierre-Louis Gauthier, "Le professeur nouveau est arrivé », Revue internationale d'éducation de Sèvres [En ligne], 21 | mars 1999, mis en ligne le 28 mai 2013, consulté le 08 janvier 2020. URL : http:// journals.openedition.org/ries/2827 ; DOI : 10.4000/ries.2827

Ce document a été généré automatiquement le 8 janvier 2020

(c) Tous droits réservés 


\title{
Le professeur nouveau est arrivé
}

\author{
Entretien avec Nicole Gaudelet, Inspectrice de l'Éducation nationale, \\ Inspection académique de l'Essonne, Evry, France \\ The new teacher has appeared. Interview with Nicole Gaudelet \\ El docente " nuevo " ha llegado. Entrevista con Nicole Gaudelet
}

\author{
Pierre-Louis Gauthier
}

La nouvelle formation initiale est-elle adaptée à la fonction enseignante d'aujourd'hui ?

Les origines personnelles et professionnelles des enseignants issus des Instituts universitaires de formation des maîtres (IUFM) composent une palette d'une grande diversité. Face à la fonction strictement enseignante, on peut dire que l'on trouve une certaine expertise dans le domaine des disciplines. Certains nouveaux professeurs sont même étonnants dans ce domaine au début de leur carrière.

Mais face au métier et sa complexité, certains d'entre eux sont démunis et n'ont aucun sens de la culture éducation nationale, ni de leurs responsabilités globales d'éducateur. Nommés " professeurs des écoles ", ils ne réalisent pas qu'ils sont aussi des instituteurs participant à "l'institution ». Par exemple, ils s'étonnent d'avoir à assurer, en dehors de leur "classe ", la surveillance de la récréation, des sorties scolaires; ils ne comprennent pas toujours qu'ils doivent tenir le registre d'appel, garant de l'obligation scolaire.

La formation en IUFM peut être confiée à des professeurs directement issus du second degré, n'ayant reçu aucune formation spécifique sur le premier degré et qui focalisent leur travail sur la didactique de leurs disciplines. Cette dérive didactique de la formation est marquée par une surproduction d'écrits professionnels, la préparation d'outils écrits tenant lieu d'outillage conceptuel. Elle pourrait être corrigée dans le cadre de la formation générale. Mais le temps est limité et les interventions magistrales sur la connaissance du système éducatif, essentiellement informatives, ne permettent pas d'aborder les vrais problèmes professionnels. Le temps limité oblige à des sacrifices: l'école maternelle n'est plus abordée systématiquement, les enseignements d'adaptation et d'intégration scolaires ne relèvent plus que du volontariat. 
La formation initiale est-elle adaptée aux réalités du terrain?

On ne prend pas suffisamment en compte, à l'TUFM, les caractéristiques et la diversité de la scolarisation dans le département ${ }^{1}$ : la présence de très nombreuses communautés nomades, l'existence d'une vaste région rurale, les communautés étrangères, le rôle des partenaires de l'école, des parents, etc.

La liaison CM2/6e qui est un maillon essentiel de la politique des cycles, n'est pas assez abordée par les formateurs. L'IUFM aurait pourtant pu être un lieu privilégié de culture partagée entre premier et second degrés. Il y a bien un stage en collège pour les professeurs des écoles et un stage en école élémentaire pour les professeurs des lycées et des collèges, ou aucun travail en commun n'est réalisé. C'est à travers les conseillers principaux d'éducation (CPE) que la rencontre espérée du primaire et du secondaire pourrait s'effectuer de manière permanente et formatrice.

La nouvelle formation s'accompagne-t-elle d'une amélioration du niveau de compétence des enseignants?

La formation en didactique a fait de sensibles progrès et permet au nouvel enseignant d'assurer des enseignements disciplinaires grâce à un outillage important, au détriment de sa vision globale du métier. L'enseignant issu de l'IUFM n'est pas formé pour travailler en équipe. Pourtant, un certain nombre d'étudiants viennent de milieux professionnels où le travail en équipe est la règle. Citons les métiers médicaux ou paramédicaux (médecins, infirmières, pharmaciens, etc.) habitués à ce mode de travail au sein de l'hôpital, que les formateurs de l'IUFM, plutôt habitués au cours magistral, n'utilisent pas suffisamment. Pourtant ces compétences pourraient constituer un point d'appui pour l'évolution du métier.

Certes il est important pour enseigner de savoir comment le savoir se construit, mais il faut aussi répondre aux besoins des élèves et à leur diversité (par exemple, la différence de développement et de réussite scolaire entre filles et garçons ${ }^{2}$ ).

Qu'est-ce que le savoir IUFM? Une série de conférences, souvent de bon niveau certes, mais détachées trop souvent d'une réflexion sur la mise en œuvre professionnelle et de tout projet collectif. Qui peut établir le lien avec la vie de la classe? Le maître formateur pourrait jouer ce rôle; ce qui demanderait que soit précisée sa place dans le programme de formation.

L'évaluation formative qui est à la base de tout processus moderne d'éducation, permettant de comprendre les sujets apprenants, de les différencier, d'expliciter leurs démarches, n'est pas encore une priorité.

\section{Quelle est l'influence de l'université sur la formation?}

L'IUFM n'a pas encore suffisamment intégré ce qui fait la spécificité de l'université : la recherche. Par contre il a accentué la tendance au cours magistral. La langue de la formation devient celle des généralités abstraites, propices aux longs débats sur la terminologie actuelle: projet, programme, préparation, compétence, etc. L'utilisation, dans la formation, des compétences antérieures des étudiants reste une préoccupation mineure.

\section{Quid de la formation continue?}

Toute formation initiale doit être une entrée dans une formation continue, une initiation à l'autoformation permanente. L'actuelle formation continue n'est pas conçue pour accompagner les équipes, en réponse aux besoins de l'évolution du 
métier. Elle s'appuie essentiellement sur les ressources, les goûts et les disponibilités des formateurs. De manière récursive, la formation continue devrait influencer la formation initiale et la ramener vers les vrais problèmes pédagogiques.

L'inspecteur d'académie et ses adjoints ont-ils la possibilité d'infléchir la formation?

L'inspecteur d'académie reste le pilote de la formation continuée et définit les axes pour le département. Les inspecteurs interviennent dans la formation. Ils sont les mieux placés pour procéder avec les équipes à une évaluation de leurs besoins de formation. Les inspecteurs s'efforcent d'accompagner les écoles et les enseignants. Mais les formateurs d'IUFM ne connaissent pas suffisamment le terrain pour adapter leur formation à ces besoins.

Avec les formateurs de l'IUFM, les acteurs du département devraient prendre en main les définitions des contenus de formation en référence aux compétences attendues. Ils peuvent accroître le professionnalisme qui fait encore défaut à la formation en IUFM, en particulier en développant une culture de l'évaluation sur la base de vrais indicateurs de la réussite des élèves et du fonctionnement de l'école, élaborés en équipe.

\section{NOTES}

1. Nicole Gaudelet est inspectrice de l'Éducation nationale auprès de l'inspecteur d'académie de l'Essonne (académie de Versailles).

2. Ségolène Royal, « La parité par l'éducation », Le Monde, 13/03/1999.

\section{RÉSUMÉS}

Dans les établissements du premier et du second degré, on a vu arriver depuis quelques années les premiers enseignants nouvellement formés dans les instituts universitaires de formation des maîtres (IUFM). Impressions des utilisateurs...

Over the past few years, France has seen the arrival in its primary and secondary schools of the first teachers to come out of the new University Institutes for Teacher Training (IUFM). User impressions...

En los centras escolares del primer y segundo grado, desde hace algunos años, se ha visto llegar a los primeras docentes recientemente formados en los institutos universitarios de formación de docentes (IUFM). Impresiones de los usuarios... 
INDEX

Index géographique : France

Mots-clés : enseignant, formation des enseignants, formation initiale, IUFM

\section{AUTEUR}

\section{PIERRE-LOUIS GAUTHIER}

Inspecteur d'académie honoraire, Paris, France 